\title{
Anti-N-Methyl-D-Aspartate Receptor Encephalitis in a Patient With Antithyroid Peroxidase Antibodies and a Parotid Pleomorphic Adenoma
}

\author{
Karlyndsay Sitterley, MD, ${ }^{1}$ Tara von Kleist, MD, ${ }^{2}$ Bridget A. Bagert, MD, MPH ${ }^{1,2}$ \\ ${ }^{1}$ The University of Queensland Faculty of Medicine, Ochsner Clinical School, New Orleans, LA ${ }^{2}$ Department of Neurology, Ochsner Clinic \\ Foundation, New Orleans, LA
}

Background: Anti-N-methyl-D-aspartate receptor (NMDA-R) encephalitis is an autoimmune disorder first described in 2007. It can cause severe neurologic and psychiatric symptoms, and patients often are misdiagnosed with a primary psychiatric disorder. Anti-NMDA-R encephalitis is an important differential diagnosis of psychosis, as the condition can be treated with the appropriate therapy.

Case Report: A 42-year-old female with a history of hypothyroidism presented for evaluation of agitation and weakness. Six months prior to her presentation at our hospital, she had complained of headache, and neuroimaging showed a small right frontal hyperintensity on fluid-attenuated inversion recovery imaging. Workup was largely unremarkable, and she improved briefly with steroids at the outside institution. She then developed psychiatric symptoms and had 4 admissions to separate inpatient psychiatric facilities. She was tried on various antipsychotic medications and mood stabilizers with no improvement and lost 30 pounds in 3 months. When the patient was transferred to our facility, her neuroimaging was stable, and serum and cerebrospinal fluid were positive for antithyroid peroxidase and NMDA-R antibodies. She was treated with intravenous immunoglobulin (IVIG), highdose steroids, and rituximab. Positron emission tomography scan revealed a parotid mass that was resected and determined to be a pleomorphic adenoma. The patient improved remarkably to her baseline and was able to return to full duties at work within 6 months. In the 2 years since her initial admission, she has had minor relapses that have responded well to rituximab and IVIG.

Conclusion: This case demonstrates a typical presentation of anti-NMDA-R encephalitis, as well as the challenges that can delay diagnosis. Symptoms vary and are often psychiatric, making these patients difficult to diagnose clinically, and they therefore require thorough investigation. This case illustrates the necessity to be inquisitive and to challenge the diagnosis when a clinical picture is not congruent because an accurate diagnosis is imperative in guiding appropriate treatment.

Keywords: Anti-N-methyl-D-aspartate receptor encephalitis, Hashimoto disease, immunomodulatory therapies, paraneoplastic syndromes

Address correspondence to Bridget A. Bagert, MD, Department of Neurology, Ochsner Clinic Foundation, 1514 Jefferson Hwy., New Orleans, LA 70121. Tel: (504) 842-3980. Email: bbagert@ochsner.org

\section{INTRODUCTION}

Anti-N-methyl-D-aspartate-receptor (NMDA-R) encephalitis is an autoimmune disorder that was first described in 2007. ${ }^{1}$ The condition is more common in women, although it may also develop in men, children, and the elderly. ${ }^{2}$ Patients often present with severe neurologic and psychiatric symptoms, including psychosis, mood disturbances, seizurelike activity, or fluctuations in consciousness. Patients are often misdiagnosed as having a primary psychiatric disorder before a proper workup is done., ${ }^{2,3}$ In females presenting with anti-NMDA-R encephalitis, a paraneoplastic ovarian teratoma is found in more than half of cases. ${ }^{4,5}$ We present a case of anti-NMDA-R encephalitis in a female with a history of hypothyroidism but without ovarian teratoma.

\section{CASE REPORT}

A 42-year-old female with a history of hypothyroidism presented to an outside emergency room with headaches described as "nonthrobbing, nonpulsating, constant, severe in the right frontal area." Magnetic resonance imaging (MRI) with and without contrast revealed a fluid-attenuated inversion recovery hyperintensity in the right frontal lobe with a small area of enhancement (these images are not available). Lumbar puncture showed lymphocytic pleocytosis but was otherwise unremarkable for infectious etiologies. 


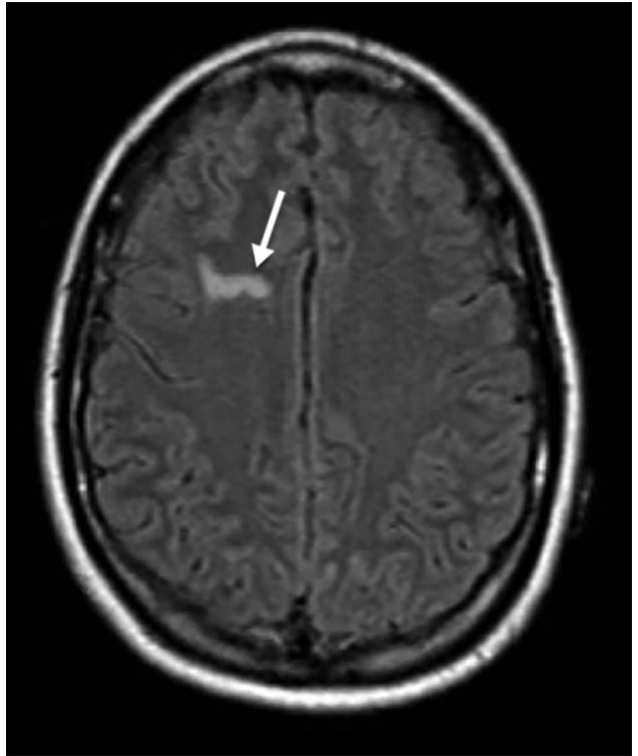

Figure 1. T1-weighted fluid-attenuated inversion recovery image precontrast showing area of hyperintensity in right frontal lobe (arrow).

Follow-up scans showed no changes and the patient was asymptomatic, so further investigations were not performed at that time.

Three months later, the patient began having uncontrollable tremors, obsessive compulsive behaviors (cleaning out closets at 10:00 pm, constantly recounting memories, pacing back and forth), catatonia, agitation, perseveration, confabulations, paranoia, and hallucinations, all of which fluctuated, and she was diagnosed with conversion disorder. In the following 3 months, she had 4 psychiatric admissions and was treated with several medication regimens including levetiracetam, lorazepam, cyclobenzaprine, escitalopram, and clonazepam with little improvement. She had an unintentional weight loss of 30 pounds during this period. Repeat MRI brain with and without contrast showed a persistent hyperintensity in the right frontal lobe but without enhancement (Figures 1 and 2). The patient was transferred to our facility 6 months after symptom onset for further evaluation and treatment.

On initial evaluation, the patient was awake but confused. She was unable to answer questions but able to follow simple commands. Her native language is Spanish; however, an interpreter provided little additional benefit to her language comprehension. Strength and sensation were intact, and reflexes were brisk throughout. She was dysarthric and apraxic.

Complete cell count and comprehensive metabolic panel were within normal limits. Thyroid-stimulating hormone was $3.095 \mathrm{ulU} / \mathrm{mL}$ (normal range, 0.4-4.000 ulU/mL), free T3 was $2.1 \mathrm{pg} / \mathrm{mL}$ (normal range, 2.3-4.2 pg/mL), and free T4 was $0.97 \mathrm{ng} / \mathrm{dL}$ (normal range, $0.71-1.51 \mathrm{ng} / \mathrm{dL}$ ). Antithyroid peroxidase antibody titers were significantly elevated at $388.2 \mathrm{IU} / \mathrm{mL}$ (normal, $<6 \mathrm{IU} / \mathrm{mL}$ ), so possible Hashimoto encephalopathy was diagnosed. A 24-hour video electroencephalogram (EEG) showed no seizures or epileptiform activity.

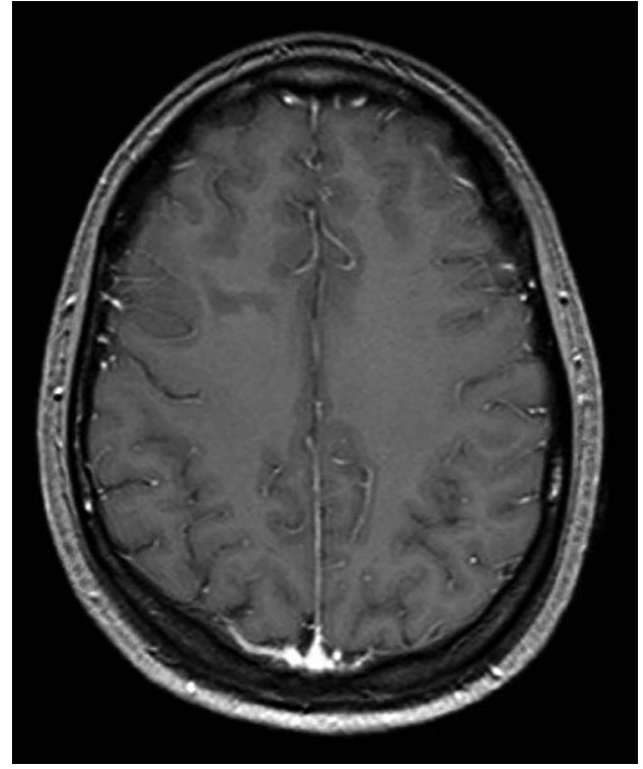

Figure 2. T1-weighted fluid-attenuated inversion recovery image postcontrast showing no area of frontal lobe hyperintensity.

Beginning on day 2 of admission, the patient was administered 1,000 mg intravenous (IV) methylprednisolone (SoluMedrol) daily for 5 days with only mild improvement. Her behavior, affect, and mental status continued to fluctuate. Serum encephalitis panel result obtained 7 days after collection was positive for NMDA-R antibodies. Repeat lumbar puncture showed elevated white blood cell count of $14 / \mathrm{cu}$ $\mathrm{mm}$ (normal range, $0-5 / \mathrm{cu} \mathrm{mm}$ ) with lymphocytosis of $94 \%$ (normal range, $40 \%-80 \%$ ), elevated protein of $56 \mathrm{mg} / \mathrm{dL}$ (normal range, $15-40 \mathrm{mg} / \mathrm{dL}$ ), and normal glycorrhachia of 42 $\mathrm{mg} / \mathrm{dL}$ (normal range, $40-70 \mathrm{mg} / \mathrm{dL}$ ). Herpes simplex virus serologies were not tested. Cerebrospinal fluid (CSF) testing confirmed the presence of NMDA-R antibodies, and the diagnosis of anti-NMDA-R encephalitis was favored over Hashimoto encephalopathy.

Transvaginal ultrasound to assess for an ovarian tumor was normal. Chest $\mathrm{x}$-ray was negative for any mass. Serum studies were negative for alpha-fetoprotein, lactate dehydrogenase, estradiol, human chorionic gonadotropin, and dehydroepiandrosterone. Beginning on day 8 of admission, the patient was administered 2 grams of IV immunoglobulin (IVIG) divided over 5 days that resulted in some improvement, but the patient continued to wax and wane and showed altered mental status, psychiatric symptoms, and confusion that persisted 10 days after the IVIG treatment. On day 20 of admission, 7 days after completing IVIG treatment, the patient was treated with $1,000 \mathrm{mg}$ IV rituximab, with a second dose of $1,000 \mathrm{mg}$ scheduled for 2 weeks after the first dose. The patient began to improve with this therapy and was discharged home after 29 days in the hospital with close follow-up.

Positron emission tomography (PET) scan 8 days following discharge revealed a focus of hypermetabolic activity in the left parotid gland. She was referred to an otolaryngologist, and the lesion was removed 2 months postdischarge. Pathology confirmed the mass to be a pleomorphic 
adenoma. Repeat transvaginal ultrasound was again negative for tumor.

The patient has shown significant improvement since her hospital discharge. She continues to receive rituximab $(1,000$ $\mathrm{mg}$ ) infusions every 6 months, with the plan to discontinue as she stabilizes. When she was discharged initially, the patient took lorazepam $1 \mathrm{mg}$ as needed every 6 hours but was gradually titrated off the medication over the course of 6 months.

The patient's husband reported that within 3 months after discharge, she was near her premorbid baseline level of function except for being more subdued, a condition he attributed to the lorazepam. Her only complaint at the 4month follow-up appointment was myalgia-possibly from prolonged steroid use, as she was maintained on $60 \mathrm{mg}$ oral prednisone therapy upon discharge with gradual taper and discontinuation over a 6-month period. Physical and neurologic examinations were otherwise unremarkable, and cognition was intact. Her appetite returned, and she regained 20 pounds. She was able to return to work as an engineer and started with half-days, with eventual graduation to her normal full-day workload 6 months after diagnosis. The patient has difficulty remembering aspects of her hospital stay and the course of her disease. In the 2 years since her initial admission, she has had minor relapses that have responded well to rituximab and IVIG.

\section{DISCUSSION}

Anti-NMDA-R encephalitis often presents with combinations of psychiatric, autonomic, and neurologic symptoms. Up to $70 \%$ of patients have a prodromal viral illness within weeks of the onset of psychiatric symptoms, although the encephalitis is not a direct result of an infectious process itself. ${ }^{6}$ Specifically, herpes simplex encephalitis has been hypothesized to be a strong prodromal illness associated with anti-NMDA-R encephalitis. ${ }^{6}$ Psychiatric symptoms include dyskinesias, ataxia, psychoses, hallucinations, echolalia, and fluctuating consciousness; many of these symptoms were seen in our patient. Up to $77 \%$ of patients ultimately diagnosed with anti-NMDA-R encephalitis were first seen by a psychiatrist and/or admitted to an inpatient psychiatric unit on initial presentation..$^{2,3,6,7}$ Results of investigations vary and include hyperintensity of a singular lesion on T2-weighted brain MRI scans; changes in EEG tracings including epileptiform activity; and CSF results with lymphocytic pleocytosis, oligoclonal bands, and increased protein. ${ }^{6}$ Of these, our patient had the behavioral symptoms, hyperintense lesion on MRI, CSF pleocytosis, and positive NMDA$R$ antibodies in CSF and serum, but she had normal EEGs throughout her hospital admission. Other investigative findings in anti-NMDA-R encephalitis include CSF elevations in proinflammatory interleukin- 6 and interleukin-17, which may explain leukocyte migration across the blood brain barrier, activation of plasma cells, and ultimately the CSF pleocytosis and intrathecal antibody production. ${ }^{6}$

NMDA-R encephalitis is often associated with a paraneoplastic syndrome. One study of 577 patients found that 220 (38\%) were associated with a neoplasm, and 94\% were ovarian teratomas. ${ }^{4}$ The remaining $6 \%$ were extraovarian tumors, including lung, breast, testis, uterus, thymus, and pancreatic neoplasms. In a study by Dalmau et al, 58 of 98 women $(59 \%)$ had an associated teratoma, with ovarian being the predominant subtype. ${ }^{5}$ Tumors most commonly occur in patients with anti-NMDA-R encephalitis who are female and between 12-45 years of age. ${ }^{4}$ With ovarian teratomas, the assessment of the tissue almost always results in the presence of NMDA-R-containing neuronal tissue that triggers the production of autoantibodies. ${ }^{5}$

Our patient did not have any evidence of an ovarian teratoma. However, PET scan revealed a mass in the left parotid gland that was confirmed to be a pleomorphic adenoma. While other masses (eg, lung, breast, testis, uterus, thymus, and pancreas) have been found in association with anti-NMDA-R encephalitis, to our knowledge, parotid pleomorphic adenoma has not been reported in the literature. ${ }^{4,8}$ This discovery and subsequent resection occurred soon after initiation of rituximab treatment. Thus, while the patient showed substantial improvement at her follow-up appointments, whether her improvement was attributable to the tumor resection or to the natural course of the disease and medical treatment is unclear. This disease often begins with a flu-like prodrome, headaches, and low-grade fever followed by the psychiatric symptoms similar to what our patient experienced-bizarre personality changes, unresponsiveness, catatonia, hallucinations-but can also include autonomic instability, cardiac dysrhythmias, seizures, coma, and death if not recognized and treated promptly. ${ }^{2-7}$

The unique psychiatric presentation of patients with antiNMDA-R encephalitis may be attributable to the widespread distribution of NMDA receptors in the central nervous system. The NMDA receptor family is a conglomerate of the NMDA receptor 1 (NR1) and NR2 subunits of glycine and glutamate receptors, respectively, and is in the hippocampus, forebrain, spinal cord, cerebellum, and basal ganglia. ${ }^{2,5}$ Especially in cases of paraneoplastic syndrome, tumor removal is often curative..$^{2,4,6,7,9-11}$ For patients without tumors, $75 \%$ can show substantial improvement with steroids, plasmapheresis, IVIG, and/or rituximab, although the progress is slower than in patients with paraneoplastic syndrome and tumor removal. ${ }^{2,11}$ However, the remaining $25 \%$ of patients without tumors are often left with neurologic deficits or die despite treatment efforts. ${ }^{2}$ The importance of early recognition is crucial because of the generally favorable response to surgical resection of the causative tumor plus medication (steroids + IVIG or plasmapheresis) or to medication only if no tumor is present. 3,6

Our patient had a history of hypothyroidism that, in conjunction with her positive antithyroid peroxidase antibodies, turned out to be a red herring, as our initial working diagnosis was Hashimoto encephalopathy. Hashimoto encephalopathy and anti-NMDA-R encephalitis present similarly with acute neuropsychiatric symptoms and occasionally with EEG and neuroimaging findings. Hashimoto encephalopathy is thought to be underdiagnosed because of its rarity and the fact that most patients do not actually have Hashimoto thyroiditis in conjunction with the psychiatric symptoms. ${ }^{12}$ Further, most patients with Hashimoto encephalopathy remain euthyroid throughout their neurologic illness and either have normal antithyroid peroxidase and antithyroglobulin antibody titers or have titers that do not correlate with the severity of the neuropsychiatric symptoms. ${ }^{9,12}$ Additionally, studies have shown that some patients with encephalitis attributable to antineuronal antibodies also have antithyroid antibodies, although the clinical significance of the 
presence of both neuronal and thyroid autoimmunity remains uncertain. ${ }^{12-14} A$ key finding in patients with Hashimoto encephalopathy is the remarkable response to steroid treatment. In one study of 251 patients with Hashimoto encephalopathy, more than $90 \%$ of patients had complete or near-complete recovery from symptoms following steroid treatment. ${ }^{15}$ Our patient's lack of response to high-dose steroid therapy cast doubt on the working diagnosis of Hashimoto encephalopathy, which was validated when serum and CSF NMDA-R antibody studies were positive.

The diagnosis of anti-NMDA-R encephalitis is reliant on antibody tests and investigations such as serum and CSF NMDA-R antibodies that may not be available at many institutions, take time to obtain from outside laboratories, or may not be present on initial investigation of the patient early in the disease course. ${ }^{2,10}$ These diagnostic challenges can lead to delays in the diagnosis of anti-NMDA-R encephalitis. Early intervention and treatment are critical, however, and can result in significant improvement. Thus, Graus et al proposed generalized diagnostic criteria that are less reliant on investigations and more reliant on clinical presentation. ${ }^{10}$ The criteria include rapid onset within less than 3 months of psychiatric or memory symptoms or altered mental status; a laboratory, clinical, or imaging finding such as CSF pleocytosis, new onset seizures, or suggestive features on $\mathrm{MRI}$; and reasonable exclusion of alternative diagnoses. ${ }^{10}$ Assessment of these criteria by a panel of experts in autoimmune encephalitides suggests improved sensitivity and specificity for the diagnosis of anti-NMDA-R encephalitis without necessitating antibody testing as before. ${ }^{10,11} \mathrm{We}$ recommend following the Graus et al criteria for any initial assessment of a patient with suspected anti-NMDA$R$ encephalitis. ${ }^{10,11}$ Considering autoimmune encephalitis in the differential diagnosis of a patient presenting with neuropsychiatric symptoms is crucial, as early diagnosis and treatment can dramatically alter a patient's long-term recovery and prognosis. ${ }^{2-4,6,11,16}$

\section{CONCLUSION}

This case highlights the challenges in the diagnosis and long-term management of a patient with autoimmune encephalitis. Remaining cognizant of autoimmune encephalitides, considering them as important differential diagnoses, and appreciating the debilitating effects that can result if they go unnoticed or untreated are critical because early treatment can prevent long-term disability.

\section{ACKNOWLEDGMENTS}

The authors have no financial or proprietary interest in the subject matter of this article.

\section{REFERENCES}

1. Dalmau J, Tüzün E, Wu H, et al. Paraneoplastic anti-N-methyl-D-aspartate receptor encephalitis associated with ovarian teratoma. Ann Neurol. 2007;6(1):25-36.

2. Wandinger KP, Saschenbrecker S, Stoecker W, Dalmau J. Anti-NMDA-receptor encephalitis: a severe, multistage, treatable disorder presenting with psychosis. J Neuroimmunol. 2011 Feb;231(1-2):86-91. doi: 10.1016/j.jneuroim.2010.09.012.

3. Sansing LH, Tüzün E, Ko MW, Baccon J, Lynch DR, Dalmau J. A patient with encephalitis associated with NMDA receptor antibodies. Nat Clin Pract Neurol. 2007 May;3(5):291-296. doi: 10.1038/ncpneuro0493.

4. Titulaer MJ, McCracken L, Gabilondo I, et al. Treatment and prognostic factors for long-term outcome in patients with anti-NMDA receptor encephalitis: an observational cohort study. Lancet Neurol. 2013 Feb;12(2):157-165. doi: 10.1016/S1474-4422(12)70310-1.

5. Dalmau J, Gleichman AJ, Hughes EG, et al. Anti-NMDA-receptor encephalitis: case series and analysis of the effects of antibodies. Lancet Neurol. 2008 Dec;7(12):1091-1098. doi: 10.1016/S1474-4422(08)70224-2.

6. Liu CY, Zhu J, Zheng XY, Ma C, Wang X. Anti-N-methyl-D-aspartate receptor encephalitis: a severe, potentially reversible autoimmune encephalitis. Mediators Inflamm. 2017;2017:6361479. doi: 10.1155/2017/6361479.

7. Kuppuswamy PS, Takala CR, Sola CL. Management of psychiatric symptoms in anti-NMDAR encephalitis: a case series, literature review and future directions. Gen Hosp Psychiatry. 2014 Jul-Aug;36(4):388-391. doi: 10.1016/j.genhosppsych.2014.02.010.

8. Çoban A, Gündoğdu G, Poyraz M, et al. NMDA receptor encephalitis with cancer of unknown primary origin. Tumori. 2016 Nov 11;102(Suppl. 2). doi: 10.5301/tj.5000447.

9. Ikura T, Katsuse O, Chiba Y, et al. Evaluation of titers of antibodies against peptides of subunits NR1 and NR2B of glutamate receptor by enzyme-linked immunosorbent assay in psychiatric patients with anti-thyroid antibodies. Neurosci Lett. 2016 Aug 15;628:201-206. doi: 10.1016/j.neulet.2016.06.028.

10. Graus F, Titulaer MJ, Balu R, et al. A clinical approach to diagnosis of autoimmune encephalitis. Lancet Neurol. 2016 Apr;15(4):391-404. doi: 10.1016/S1474-4422(15)00401-9.

11. Ho ACC, Mohammad SS, Pilla SC, et al. High sensitivity and specificity in proposed clinical diagnostic criteria for anti-N-methyl-D-aspartate receptor encephalitis. Dev Med Child Neurol. 2017 Dec;59(12):1256-1260. doi: 10.1111/dmcn.13579.

12. de Holanda NC, de Lima DD, Cavalcanti TB, Lucena CS, Bandeira F. Hashimoto's encephalopathy: systematic review of the literature and an additional case. J Neuropsychiatry Clin Neurosci. 2011 Fall;23(4):384-390. doi: 10.1176/jnp.23.4.jnp384.

13. Kishitani $T$, Matsunaga $A$, Ikawa $M$, et al. Limbic encephalitis associated with anti-NH2-terminal of $\alpha$-enolase antibodies: a clinical subtype of Hashimoto encephalopathy. Medicine (Baltimore). 2017 Mar;96(10):e6181. doi: 10.1097/MD.0000000000006181.

14. Tüzün E, Erdağ E, Durmus $H$, et al. Autoantibodies to neuronal surface antigens in thyroid antibody-positive and -negative limbic encephalitis. Neurol India. 2011 Jan-Feb;59(1):47-50. doi: 10.4103/0028-3886.76857.

15. Laurent C, Capron J, Quillerou B, et al. Steroid-responsive encephalopathy associated with autoimmune thyroiditis (SREAT): characteristics, treatment and outcome in 251 cases from the literature. Autoimmun Rev. 2016;15(12):1129-1133.

16. Zhang W, Yan L, Jiao J. Repeated misdiagnosis of a relapsed atypical anti-NMDA receptor encephalitis without an associated ovarian teratoma. Neurosci Lett. 2017 Jan 18;638:135-138. doi: 10.1016/j.neulet.2016.11.057.

This article meets the Accreditation Council for Graduate Medical Education and the American Board of Medical Specialties Maintenance of Certification competencies for Patient Care and Medical Knowledge. 